\title{
GMR
}

\section{Development of retrotransposon-based markers IRAP and REMAP for cassava (Manihot esculenta)}

\author{
B.C. Kuhn ${ }^{1}$, C.A. Mangolin ${ }^{2}$, E.R. Souto ${ }^{3}$, C.M. Vicient ${ }^{4}$ and M.F.P.S. Machado ${ }^{5}$ \\ ${ }^{1}$ Programa de Pós-Graduação em Genética e Melhoramento, \\ Universidade Estadual de Maringá, Maringá, PR, Brasil \\ 2Departamento de Biologia Celular e Genética, Universidade Estadual de Maringá, \\ Maringá, PR, Brasil \\ ${ }^{3}$ Departamento de Agronomia, Universidade Estadual de Maringá, Maringá, Brasil \\ ${ }^{4}$ Departamento de Genética Molecular, \\ Centro de Pesquisa em Agrogenômica (CSIC-IRTA-UAB-UB), Campus UAB, \\ Bellaterra (Cerdanyola del Vallès), Barcelona, Espanha \\ ${ }^{5}$ Departamento de Biologia Celular e Genética, Universidade Estadual de Maringá, \\ Maringá, PR, Brasil \\ Corresponding author: B.C. Kuhn \\ E-mail: bettybio@hotmail.com
}

Genet. Mol. Res. 15 (2): gmr.15027149

Received November 30, 2015

Accepted January 18, 2016

Published April 7, 2016

DOI http://dx.doi.org/10.4238/gmr.15027149

ABSTRACT. Retrotransposons are abundant in the genomes of plants. In the present study, inter-retrotransposon amplified polymorphism (IRAP) and retrotransposon-microsatellite amplified polymorphism (REMAP) markers were developed for the cassava genome (Manihot esculenta Crantz). Four cassava cultivars (Fécula Branca, IPR-União, Olho Junto, and Tamboara, two samples per cultivar) were used to obtain IRAP and REMAP fingerprints. Twelve designed primers were amplified alone and in combinations. The 42 IRAP/REMAP primer combinations amplified 431 DNA segments (bands; markers) of which $36(8.36 \%)$ were polymorphic. The largest number of informative markers (16) was detected using the 
primers AYF2 and AYF2xAYF4. The number of bands for each primer varied from 3 to 16 , with an average of 10.26 amplified segments per primer. The size of the amplified products ranged between 100 and 7000 bp. The AYF2 primer generated the highest number of amplified segments and showed the highest number of polymorphic bands $(68.75 \%)$. Two samples of each cassava cultivar were used to illustrate the usefulness and the polymorphism of IRAP/REMAP markers. IRAP and REMAP markers produced a high number of reproducible bands, and might be informative and reliable for investigation of genetic diversity and relationships among cassava cultivars.

Key words: Retrotransposons; Cassava; Diversity; Primer design

\section{INTRODUCTION}

Cassava (Manihot esculenta Crantz) is native to South America and was domesticated about 8000 years ago (Bull et al., 2011). The tuberous roots of cassava have high starch content and are an important source of carbohydrate (Mejía-Agüero et al., 2012). Cassava is one of the most important crops worldwide since it is the predominant food in the staple diet of several countries in Africa, Asia, and Latin America (McMahon et al., 1995). It is also used for animal feed and as raw material for starch production (Wongtiem et al., 2011). Cassava is also important for activities not associated with food, and is used in textile and paper industries (Mejía-Agüero et al., 2012), for ethanol production, and as a biofuel (Wongtiem et al., 2011). Thus, knowledge on the genetic diversity and relationships among cassava cultivars is an important requisite to improve and increase their production.

Since there are few reliable phenotypic morphological characteristics in the Manihot genus (Olsen and Schaal, 2001), molecular markers have been used to infer the genetic relationships among cassava cultivars. Several molecular markers, such as isozymes (Resende et al., 2004), randomly amplified polymorphic DNA (RAPD) (Zacarias et al., 2004), amplified fragment length polymorphisms (AFLP) (Chavarriaga-Aguirre et al., 1999), simple sequence repeats (SSR) (Chavarriaga-Aguirre et al., 1999; Siqueira et al., 2009; Lekha et al., 2010), and simple sequence repeats from expressed sequence tags (EST-SSR) (Kunkaew et al., 2011; Sraphet et al., 2011), have been used to analyze the genetic diversity and relationships among cassava cultivars. However, retrotransposon markers, which are highly promising for phylogenetic studies, gene mapping, and genetic diversity (Kalendar et al., 1999), have not yet been analyzed in cassava cultivars.

Retrotransposons are the most abundant transposable elements present in eukaryotic genomes and are classified into long terminal repeats (LTRs) and non-LTRs (Kumar and Bennetzen, 1999). Retrotransposons are dispersed throughout plant genomes and some retrotransposon families are represented by thousands of copies (Kalendar et al., 2011). New copies of retrotransposons are randomly inserted into preexisting sequences of the genome via a copy-paste system, which consequently increases the copy number (Kalendar and Schulman, 2006). Retrotransposons contribute to the size, structure, variation, and diversity of the genome. In addition, they greatly effect gene function and cover a high percentage of the genome (Gbadegesin and Beeching, 2010).

Sequence-specific amplification polymorphisms (S-SAPs) were the first molecular markers based on retrotransposons to be developed. These involve a primer annealing to the end of the retrotransposon and a site corresponding to an AFLP restriction enzyme (Waugh et al.. 1997). 
Two other systems of retrotransposon-based molecular markers, inter-retrotransposon amplified polymorphism (IRAP) and retrotransposon-microsatellite amplified polymorphism (REMAP), were also developed by Kalendar et al. (1999). IRAP markers involve the PCR amplification of DNA sequences between two nearby retrotransposons, using a primer designed from the LTR sequence of a retrotransposon. On the other hand, REMAP uses a primer based on the LTR sequence and an SSR primer with an anchored nucleotide (non-SSR) at the 3 ' end of the primer.

A total of 154 transposable elements of the cassava genome were partially sequenced by Gbadegesin et al. (2008), including 59 families of Ty1/copia, 26 families of Ty3/gypsy retrotransposons, and 40 families of $\mathrm{En} / \mathrm{Spm}$ transposons. The cassava genome has been sequenced, but to our knowledge, no primers have been designed for IRAP and REMAP markers in order to study this species. Therefore, in the current study, IRAP and REMAP molecular markers specific for the cassava genome were developed.

\section{MATERIAL AND METHODS}

\section{Plant material and DNA isolation}

The cassava varieties 'Fécula Branca' and 'Olho Junto' (with low cyanogenic acid content), 'IPR-União' and 'Tamboara' (with high cyanogenic acid content) were used in the present study (Table 1). The four cassava cultivars are cultivated the most in the state of Paraná, Brazil, and were obtained from the IAPAR (Agronomic Institute of Paraná - Brazil) germplasm bank.

Table 1. Cassava cultivars, content of cyanogenic acid and utility of the four cultivars (IAPAR - Instituto Agronômico
do Paraná).
\begin{tabular}{l|l|l}
\hline Cultivar & Cyanogenic acid & Utility \\
\hline Fécula Branca & Low & Immediate human consumption \\
\hline IPR-União & High & Industry \\
\hline Olho Junto & High & Immediate human consumption \\
\hline Tamboara & High & Industry \\
\hline
\end{tabular}

Total DNA was isolated from samples of each cultivar using the cetyltrimethylammonium bromide (CTAB) protocol described by Knapp and Chandlee (1996), and adapted by Carvalho (Cenargen - Embrapa). Genomic DNA was dissolved in TE (1 mM EDTA, 10 mM Tris-HCl, pH 8.0) and quantified using a picodrop (Pico200 spectrophotometer).

\section{Primer design}

Primers for the LTR sequences of the IRAP and REMAP markers were designed from the cassava sequence database, while primers for the SSR sequences of the REMAP markers were randomly designed and further tested to validate their utility. Retrotransposon sequences from three families of transposable elements in the cassava genome have been described (Gbadegesin et al., 2008) and deposited in GenBank under accession No. AY946045-AY946199. The retrotransposon sequences obtained from GenBank were submitted to Phytozome Blast (http://www.phytozome.net) to find the adjacent sequences of the retrotransposon. The adjacent sequences of the retrotransposon were then analyzed by the LTR finder program for the LTR 
sequences. Twelve different LTR sequences were obtained and aligned using CLUSTAL W (Thompson et al., 1994). The designed primers were based on the most conserved region for each LTR with amplification directed outwards from the retrotransposon (Kalendar and Schulman, 2006). One or two LTR primers were used in the same reaction for the IRAP markers (Figure 1), whereas one LTR primer and one SSR primer (with two or three repeated selective bases) were used for the REMAP markers (Figure 2).

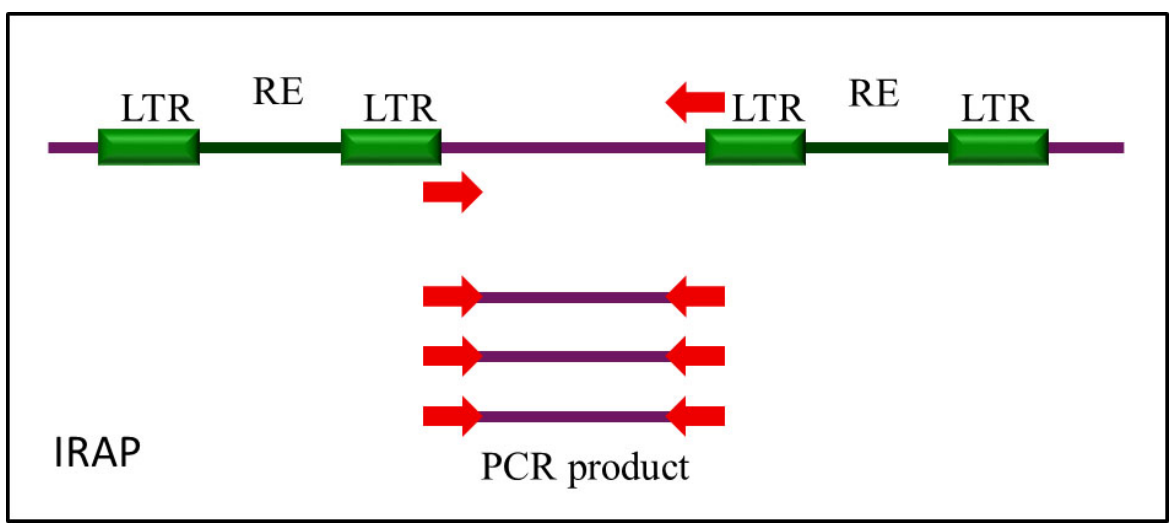

Figure 1. Product from IRAP amplification. IRAP: The primer amplified the sequence between two retrotransposons. LTR: Long Terminal Repeat; RE: Retrotransposon. Modified from Kalendar and Schulman (2006).

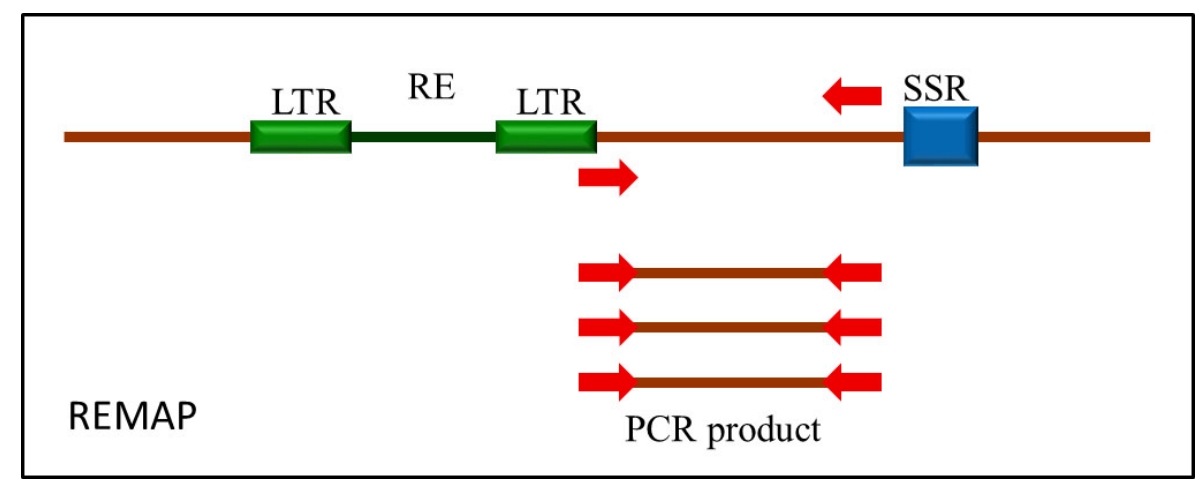

Figure 2. Product from REMAP amplification. REMAP: The primer amplified the sequence between a retrotransposon and a SSR sequence. LTR: Long Terminal Repeat; RE: Retrotransposon; SSR: Simple Sequence Repeat. Modified from Kalendar and Schulman (2006).

\section{IRAP and REMAP amplification}

PCRs were performed in a 25- $\mu \mathrm{L}$ volume containing: 30 ng DNA, 1X PCR buffer [75 mM Tris- $\mathrm{HCl}, \mathrm{pH}$ 9.0, $50 \mathrm{mM} \mathrm{KCl}, 20 \mathrm{mM}\left(\mathrm{NH}_{4}\right)_{2} \mathrm{SO}_{4}$ ], $2.5 \mathrm{mM} \mathrm{MgCl}_{2}, 4 \mathrm{pM}$ each primer, $100 \mu \mathrm{L} \mathrm{dNTP}$, and $1 \mathrm{U}$ PrimeSTAR DNA polymerase (Takara Bio, Tokyo, Japan). Amplifications were performed in a MJ Research PTC-200 PCR Peltier Thermal Cycler (Bio-Rad, Hercules, USA). After 10 min of initial denaturation at $95^{\circ} \mathrm{C}$, amplifications were carried out for 36 cycles at $94^{\circ} \mathrm{C}$ for $30 \mathrm{~s}$; at $55^{\circ} \mathrm{C}$ for $30 \mathrm{~s}$, and at $72^{\circ} \mathrm{C}$ for $3 \mathrm{~min}$, with a final extension of $10 \mathrm{~min}$ at $72^{\circ} \mathrm{C}$. The PCR products 
were identified by electrophoresis in 3\% agarose (ECOGEN, AG-0600, Madrid, Spain) in 1X TAE buffer followed by EtBr staining and UV visualization. PCRs were repeated in triplicate and similar results were obtained. Each primer was tested for efficiency in the yield of IRAP segments and for fingerprint quality (separate and well defined bands in the gel).

\section{Data scoring and analysis}

IRAP and REMAP bands were scored 1 for the presence or 0 for the absence of bands. Binary matrices (presence/absence) were prepared from IRAP and REMAP products. Each PCR product represented a single locus. Monomorphic bands were removed from the raw scored data sets. Weakly stained bands were not scored. The Dice coefficient was employed to estimate genetic similarity. The binary data obtained were then analyzed with NTSYSpc 2.1 program (Rohlf, 1989), which generated the genetic distance matrix (Nei and Li, 1979) and drew the UPGMA (Unweighted pair group method with arithmetic mean) dendrogram.

\section{RESULTS AND DISCUSSION}

\section{Development of IRAP for cassava germplasm genotyping}

Twelve LTR retrotransposon families were identified from the cassava partial sequences deposited in databases using the LTR finder. Several LTR sequences were obtained for each family and aligned with ClustalW (Thompson et al. 1994). The most conserved regions were used to design primers containing 21-24 bp with a high $\mathrm{C}: \mathrm{G}$ proportion. Primers were located next to the end of the retrotransposon element facing out from the LTR (Table 2). Twelve retrotransposon primers were designed and all primers were used for the analysis, alone or in combinations.

Table 2. Selected primers used to generate IRAP and REMAP in cassava.

\begin{tabular}{|c|c|c|c|}
\hline Name & Class & Primer & $\% \mathrm{GC}$ \\
\hline AYF1 & LTR & 3'-GGATCCTAGCGCCGGTAGCGGT-5' & 68.18 \\
\hline AYF2 & LTR & 3'-CTCTCTGTACGCTCCTGTTCGTAC-5' & 54.17 \\
\hline AYF3 & LTR & 3'-ACGGCTTCGACAACCGCTTATC-5' & 54.55 \\
\hline AYF4 & LTR & 3'-GACCGGCTTCGACAACCGCGAGTC-5' & 66.67 \\
\hline AYF5 & LTR & 3'-CCTAGCGCCGGTAGCGGTCCGA-5' & 72.72 \\
\hline AYF6 & LTR & 3'-CCGGAGTCTAGCCGGGTATTAC-5' & 59.09 \\
\hline AYF7 & LTR & 3'-GAACAAGTTATGCTATTATGCA-5' & 31.81 \\
\hline AYF8 & LTR & 3'-CCAAGGCTAGTTGCTCCATGTC-5' & 54.54 \\
\hline AYF9 & LTR & 3'-AGTCTCTAATAGGCTGGGGTTC-5' & 50.00 \\
\hline AYF10 & LTR & 3'-CAAATATCTTATGTGATAGAG -5' & 28.57 \\
\hline AYF11 & LTR & 3'-CATTGAAGATATAACTTATCCT-5' & 27.27 \\
\hline AYF12 & LTR & 3'-CTTCTCTCTCTGTACGCTCCTG-5' & 54.54 \\
\hline
\end{tabular}

Each LTR primer was tested alone and with all possible combinations for IRAP markers. In the case of REMAP, four LTR primers were combined with four SSR primers. The scoring criteria involved the number, intensity, and sharpness of the PCR products, as well as the degree of polymorphism among the genotypes. Seventy-eight combinations of LTR primers were used in one cassava sample and 53 sharp bands were observed in the gel (49 IRAP and 4 REMAP) so that applicability of the selected markers could be explored. The patterns of amplification of the different primer combinations were initially analyzed using genomic DNA extracted from two samples of each cassava cultivar and all primer combinations that produced bands were used 
to amplify the DNA from the four cassava cultivars (Fécula Branca, Olho Junto, IPR-União, and Tamboara). All combinations of primers tested that resulted in poor amplification or few products were discarded from the analysis. The 42 primer combinations used in the analysis involved 40 IRAP and 2 REMAP (Table 3).

Table 3. Combinations of primers used to generate IRAP and REMAP markers in cassava showing the total of amplified segments (TAS) as well as the number of polymorphic amplified segments (PAS).

\begin{tabular}{|c|c|c|c|c|c|}
\hline Primer(s) & TAS & PAS & Primer(s) & TAS & PAS \\
\hline AYF1 & 10 & & AYF2xAYF8 & 14 & \\
\hline AYF2 & 16 & 11 & AYF2xAYF9 & 13 & 1 \\
\hline AYF4 & 15 & 1 & AYF3xAYF4 & 7 & \\
\hline AYF5 & 9 & & AYF3XAYF5 & 5 & \\
\hline AYF8 & 15 & 4 & AYF3xAYF8 & 13 & 3 \\
\hline AYF9 & 10 & 2 & AYF3xAYF9 & 15 & 1 \\
\hline AYF12 & 12 & & AYF4xAYF5 & 9 & \\
\hline AYF1xAYF2 & 13 & 1 & AYF4XAYF7 & 14 & \\
\hline AYF1xAYF3 & 12 & 4 & AYF4xAYF8 & 13 & \\
\hline AYF1xAYF4 & 7 & & AYF4XAYF9 & 14 & \\
\hline AYF1xAYF5 & 12 & 1 & AYF4xAYF11 & 9 & \\
\hline AYF1xAYF6 & 3 & & AYF4xAYF12 & 7 & \\
\hline AYF1xAYF8 & 10 & 2 & AYF5xAYF6 & 4 & \\
\hline AYF1xAYF9 & 10 & & AYF5XAYF8 & 9 & \\
\hline AYF1XAYF10 & 9 & & AYF5XAYF9 & 11 & 1 \\
\hline AYF1XAYF11 & 9 & & AYF5xAYF10 & 10 & 1 \\
\hline AYF1XAYF12 & 11 & & AYF5xAYF11 & 9 & 1 \\
\hline AYF2xAYF3 & 12 & 2 & AYF8xAYF9 & 12 & \\
\hline AYF2xAYF4 & 16 & & AYF9xAYF12 & 8 & \\
\hline AYF2xAYF5 & 7 & & AG8 xAYF1 & 8 & \\
\hline \multirow[t]{2}{*}{ AYF2xAYF6 } & 3 & & AC8 xAYF5 & 6 & \\
\hline & & & Total & 431 & 36 \\
\hline
\end{tabular}

The 42 IRAP/REMAP primer combinations amplified 431 DNA segments (bands; markers) of which $36(8.36 \%)$ were polymorphic (Table 3$)$. The total number of bands for each primer varied between 3 and 16, with an average of 10.26 amplified segments per primer. The size of amplified products ranged between 100 and 7000 bp. Primers AYF2 and AYF2xAYF4 produced the largest number of bands (16 bands). Fifteen of the 42 primer combinations tested produced polymorphic bands and were therefore useful to investigate genetic variability in the cassava genome, and to establish genetic relationships among cassava cultivars. The largest number of informative markers was detected using the primer AYF2 (11 bands, $68.75 \%$ of the total amplified), while the primer combinations AYF8 and AYF1XAYF3 produced four polymorphic bands (Figure 3).

Figure 3 shows that the AYF2 primer was also used to characterize intra cultivar polymorphism in cassava. The polymorphism evident in only two samples of the IPR União cultivar (Figure 3) indicated that the IRAP/REMAP markers may be useful for investigating the genetic variability within cassava cultivars. Primers AYF4, AYF8, AYF3XAYF8, and AYF5XAYF10 also revealed polymorphism within Tamboara and Fécula Branca cultivars (results not shown).

\section{Genetic diversity among cassava cultivars using IRAP/REMAP makers}

The percentage of polymorphic segments ranged from $1.21 \%$ in the IPR União and Fécula Branca samples (two IRAP/REMAP markers) to $4.85 \%$ in the Tamboara sample (eight IRAP/REMAP markers). In the Olho Junto samples, the percentage of polymorphic segments was zero (polymorphism was absent) and the genetic distance among the four samples was 0.078. The UPGMA dendrogram, obtained from the cluster analysis of Nei's unbiased genetic distance, 
revealed one main group comprising the cassava cultivars IPR União, Fécula Branca, and Tamboara, whereas samples of Olho Junto formed an isolated group (Figure 4). The value of Nei's identity (I) varied from 0.8567 (between the Olho Junto and Tamboara samples) to 0.9431 (between Fécula Branca and Tamboara samples) (Table 4). These results suggest that IPR União, Fécula Branca, and Tamboara are grouped together and Olho Junto is part of a different group (Figure 4).

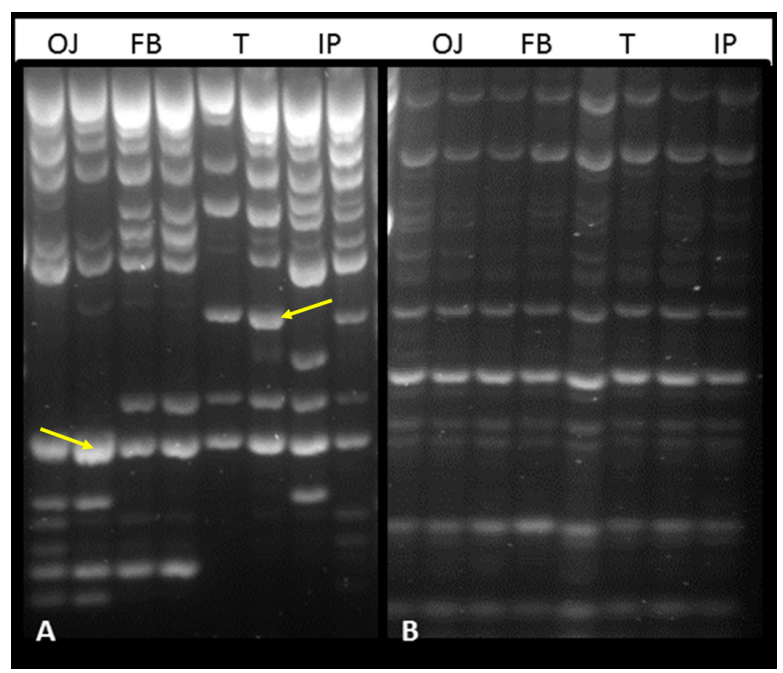

Figure 3. IRAP gels from the four cassava cultivars, showing the polymorphism. A. primer AYF2 (11 polymorphic bands); B. AYF8 (4 polymorphic bands). Samples: OJ = Olho Junto, FB = Fécula Branca, $T=$ Tamboara, IP = IPRUnião. Arrow in the left indicates monomorphic amplified band, arrow in the right indicates polymorphic amplified band.

Table 4. Nei's genetic identity (above diagonal) and genetic distance (below diagonal).

\begin{tabular}{|c|c|c|c|c|}
\hline Variety & Olho Junto & Fécula Branca & Tamboara & IPR União \\
\hline Olho Junto & 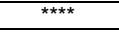 & 0.890 & 0.856 & 0.873 \\
\hline Fécula Branca & 0.115 & $* * * *$ & 0.943 & 0.934 \\
\hline Tamboara & 0.154 & 0.058 & 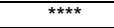 & 0.935 \\
\hline IPR União & 0.135 & 0.068 & 0.066 & 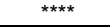 \\
\hline
\end{tabular}

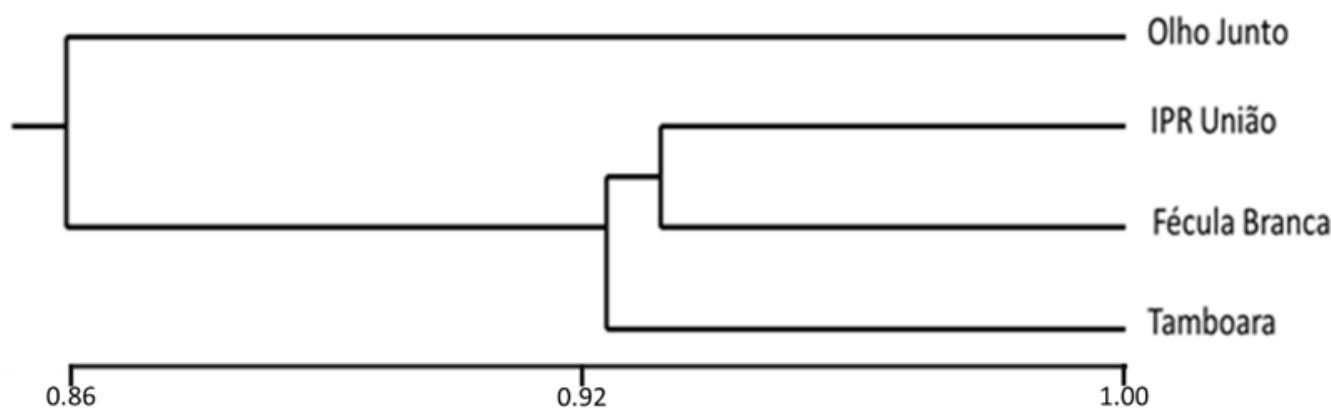

Figure 4. Dendrogram from four cassava cultivars. An unweighted pair-group method with arithmetic averages (UPGMA) cluster analysis based on genetic distances of Nei and Li (1979) obtained from the IRAP and REMAP primers, among cassava cultivars. 
The cassava genome was estimated to be about $772 \mathrm{Mbp}$ in size. A total of 59 families of transposable elements were detected including Ty $1 /$ copia, as well as 26 Ty3/gypsy retrotransposons and $40 \mathrm{En} / \mathrm{Spm}$ transposons (Gbadegesin et al., 2008). Furthermore, the abundance of LTR retrotransposons in the cassava genome was used to develop primers for the IRAP and REMAP marker systems. The IRAP and REMAP marker systems have previously been used to study genetic diversity in Helianthus (Vukich et al., 2009), flax (Smýkal et al., 2011), and maize (Kuhn et al., 2014). They have also been used to analyze the genetic diversity in cassava. In-depth knowledge on the genetic diversity in cassava cultivars is highly important for their further use in breeding programs and for conservation in germplasm banks.

In the present study, the abundance of retrotransposon LTRs in cassava was confirmed, since 431 amplified DNA segments (molecular markers) were obtained using 42 IRAP and REMAP primer combinations. The AYF3, AYF6, AYF7, AYF10, and AYF11 primers failed to amplify any band when they were used alone, but they generated bands when used in combination with other primers (Table 3 ). The number of IRAP/REMAP markers obtained in the current study (431) using only two samples each of Olho Junto, Fecula Branca, IPR União, and Tamboara was higher than the number of molecular markers used in other studies of the cassava genome. Molecular markers, such as isozymes (22) (Chavarriaga-Aguirre et al., 1999); (82) (Resende et al., 2004), RAPD markers (311) (Zacarias et al., 2004), SSR markers (43) (Chavarriaga-Aguirre et al., 1999); (46) (Siqueira et al, 2009), and EST-SSR markers (425) (Kunkaew et al. 2011) produced the lowest number of reproducible bands. Although S-SAP is a co-dominant marker retrotransposon, the enzymatic steps may increase the possibility of generating impurities and incorrect digestion (Kalendar and Schulman, 2006). When compared to other DNA markers, IRAP and REMAP are simple techniques, easy to operate, and produce a high number of reproducible bands. They may be informative and reliable for the study of genetic diversity and relationships among cassava cultivars. Only two samples from each cassava cultivar were used in the current analysis to illustrate the usefulness and polymorphism of the IRAP/REMAP markers.

Estimates of polymorphism, genetic distance, and genetic identity are illustrative and may not be used to provide conclusive information on the genetic diversity among the four cultivars analyzed. The number of cultivars (four) and samples of each cultivar analyzed were low (two samples of each cultivar), making it difficult to group them based on different levels of cyanogenic acid. However, a larger number of samples of each cultivar Olho Junto, Fécula Branca, IPR União, and Tamboara, as well as other cassava cultivars, will be analyzed using the IRAP/ REMAP markers to investigate the genetic diversity and relationships among them, and to detect correlations between phenotypic characteristics and retrotransposon markers. Knowledge on the genetic diversity and relationships among cassava cultivars is an important requisite to improve and increase the production of different cultivars.

The IRAP and REMAP primers designed in this study were effective at assessing cassava diversity. The retrotransposon-based marker was efficient at differentiating the four cassava cultivars and for determining diversity within the samples. These molecular markers may be useful tools for application in breeding and in the analysis of diversity of cassava and another organisms.

\section{Conflicts of interest}

The authors declare no conflict of interest. 


\section{ACKNOWLEDGMENTS}

This project was carried out in the Centre for Research in Agricultural Genomics CRAG, Spain (CSIC-IRTA-UAB-UB), and was supported by CAPES (Coordenação de Aperfeiçoamento Pessoal de Nível Superior), Brazil.

\section{REFERENCES}

Bull SE, Ndunguru J, Gruissem W, Beeching JR, et al. (2011). Cassava: constraints to production and the transfer of biotechnology to African laboratories. Plant Cell Rep. 30: 779-787. http://dx.doi.org/10.1007/s00299-010-0986-6

Chavarriaga-Aguirre CP, Maya M, Tohme J, Duque MC, et al. (1999). Using microsatellites, isozymes and AFLPs to evaluate genetic diversity and redundancy in the cassava core collection and to assess the usefulness of DNA-based markers to maintain germplasm collections. Mol. Breed. 5: 263-273. http://dx.doi.org/10.1023/A:1009627231450

Gbadegesin MA and Beeching JR (2010). Enhancer/Suppressor mutator (En/Spm)-like transposable elements of cassava (Manihot esculenta) are transcriptionally inactive. Genet. Mol. Res. 9: 639-650. http://dx.doi.org/10.4238/vol9-2gmr713

Gbadegesin MA, Wills MA and Beeching JR (2008). Diversity of LTR-retrotransposons and Enhancer/Suppressor Mutatorlike transposons in cassava (Manihot esculenta Crantz). Mol. Genet. Genomics 280: 305-317. http://dx.doi.org/10.1007/ s00438-008-0366-x

Kalendar R and Schulman AH (2006). IRAP and REMAP for retrotransposon-based genotyping and fingerprinting. Nat. Protoc. 1: 2478-2484. http://dx.doi.org/10.1038/nprot.2006.377

Kalendar R, Grob T, Regina M, Souniemi A, et al. (1999). IRAP and REMAP: two new retrotransposon-based DNA fingerprinting techniques. Theor. Appl. Genet. 98: 704-711. http://dx.doi.org/10.1007/s001220051124

Kalendar R, Flavell AJ, Ellis THN, Sjakste T, et al. (2011). Analysis of plant diversity with retrotransposon-based molecular markers. Heredity (Edinb) 106: 520-530. http://dx.doi.org/10.1038/hdy.2010.93

Knapp JE and Chandlee JM (1996). RNA/DNA mini-prep from a single sample of orchid tissue. Biotechniques 21: 54-56.

Kuhn BC, López-Ribera I, da Silva Machado MdeF and Vicient CM (2014). Genetic diversity of maize germplasm assessed by retrotransposon-based markers. Electrophoresis 35: 1921-1927. http://dx.doi.org/10.1002/elps.201400038

Kumar A and Bennetzen JL (1999). Plant retrotransposons. Annu. Rev. Genet. 33: 479-532. http://dx.doi.org/10.1146/annurev. genet.33.1.479

Kunkaew S, Yoocha T, Sraohet S, Boonchanawiwat A, et al. (2011). Construction of a genetic linkage map using simple sequence repeat markers from expressed sequence tags for cassava (Manihot esculenta Crantz). Mol. Breed. 27: 67-75. http://dx.doi.org/10.1007/s11032-010-9414-4

Lekha SS, Pillai VS and Kumar JS (2010). Molecular genotyping of Indian cassava cultivars using SSR markers. Adv. Environ. Biol. 4: 224-233.

McMahon JM, White WLB and Sayre RT (1995). Cyanogenesis in cassava (Manihot esculenta Crantz). J. Exp. Bot. 46: 731741. http://dx.doi.org/10.1093/jxb/46.7.731

Mejía-Agüero LE, Galeno F, Hernández-Hernández O, Matehus J, et al. (2012). Starch determination, amylose content and susceptibility to in vitro amylolysis in flours from the roots of 25 cassava varieties. J. Sci. Food Agric. 92: 673-678. http:// dx.doi.org/10.1002/isfa.4629

Nei M and Li WH (1979). Mathematical model for studying genetic variation in terms of restriction endonucleases. Proc. Natl. Acad. Sci. USA 76: 5269-5273. http://dx.doi.org/10.1073/pnas.76.10.5269

Olsen K and Schaal B (2001). Microsatellite variation in cassava (Manihot esculenta, Euphorbiaceae) and its wild relatives: further evidence for a southern Amazonian origin of domestication. Am. J. Bot. 88: 131-142. http://dx.doi.org/10.2307/2657133

Resende AG, Filho PSV and Machado MFPS (2004). Esterase polymorphism marking cultivars of Manihot esculenta, Crantz. Braz. Arch. Biol. Technol. 47: 347-353. http://dx.doi.org/10.1590/S1516-89132004000300003

Rohlf FJ (1989) NTSYS-pc Numerical taxonomy and multivariate analysis system, version 1.50. Setauket, NY: Exel Software.

Siqueira MV, Queiroz-Silva JR, Bressan EA, Borges A, et al. (2009). Genetic characterization of cassava (Manihot esculenta) landraces in Brazil assessed with simple sequence repeats. Genet. Mol. Biol. 32: 104-110. http://dx.doi.org/10.1590/ $\underline{\text { S1415-47572009005000010 }}$

Smýkal P, Bačová-Kerteszová N, Kalendar R, Corander J, et al. (2011). Genetic diversity of cultivated flax (Linum usitatissimum L.) germplasm assessed by retrotransposon-based markers. Theor. Appl. Genet. 122: 1385-1397. http://dx.doi. org/10.1007/s00122-011-1539-2

Sraphet S, Boonchanawiwat A, Thanyasiriwat T, Boonseng O, et al. (2011). SSR and EST-SSR-based genetic linkage map of cassava (Manihot esculenta Crantz). Theor. Appl. Genet. 122: 1161-1170. http://dx.doi.org/10.1007/s00122-010-1520-5 
Thompson JD, Higgins DG and Gibson TJ (1994). CLUSTAL W: improving the sensitivity of progressive multiple sequence alignment through sequence weighting, position-specific gap penalties and weight matrix choice. Nucleic Acids Res. 22 : 4673-4680. http://dx.doi.org/10.1093/nar/22.22.4673

Vukich M, Schulman AH, Giordani T, Natali L, et al. (2009). Genetic variability in sunflower (Helianthus annuus L.) and in the Helianthus genus as assessed by retrotransposon-based molecular markers. Theor. Appl. Genet. 119: 1027-1038. http:// dx.doi.org/10.1007/s00122-009-1106-2

Waugh R, McLean K, Flavell AJ, Pearce SR, et al. (1997). Genetic distribution of Bare-1-like retrotransposable elements in the barley genome revealed by sequence-specific amplification polymorphisms (S-SAP). Mol. Gen. Genet. 253: 687-694. http://dx.doi.org/10.1007/s004380050372

Wongtiem P, Courtois D, Florin B, Juchaux M, et al. (2011). Effects of cytokinins on secondary somatic embryogenesis of selected clone Rayong 9 of Manihot esculenta Crantz for ethanol production. Afr. J. Biotechnol. 10: 1600-1608.

Zacarias AM, Botha AM, Labuschagne MT and Benesi IRM (2004). Characterization and genetic distance analysis of cassava (Manihot esculenta Crantz) germplasm from Mozambique using RAPD fingerprinting. Euphytica 138: 49-53. http://dx.doi. org/10.1023/B:EUPH.0000047057.05642.74 